\title{
PMF Regimen
}

National Cancer Institute

\section{Source}

National Cancer Institute. PMFRegimen. NCI Thesaurus. Code C161993.

A chemotherapy regimen consisting of fluorouracil, melphalan, and methotrexate that

may be used in the treatment of advanced breast and ovarian cancers. 